Please quote as: Traumer, F.; Oeste-Reiß, S. \& Leimeister, J. M. (2017): Towards a Future Reallocation of Work between Humans and Machines - Taxonomy of Tasks and Interaction Types in the Context of Machine Learning. In: International Conference on Information Systems (ICIS). Seoul, South Korea. 


\title{
Towards a Future Reallocation of Work between Humans and Machines - Taxonomy of Tasks and Interaction Types in the Context of Machine Learning
}

\author{
Short Paper
}

\author{
Fabian Traumer \\ Information Systems, \\ University of Kassel \\ fabian.traumer@wi-kassel.de
}

\author{
Sarah Oeste-Reiß \\ Information Systems, \\ University of Kassel \\ oeste-reiss@uni-kassel.de
}

\author{
Jan Marco Leimeister \\ Information Systems, \\ University of Kassel, \\ leimeister@uni-kassel.de \\ Institute of Information \\ Management, \\ University of St. Gallen \\ janmarco.leimeister@unisg.ch
}

\begin{abstract}
In today's race for competitive advantages, more and more companies implement innovations in artificial intelligence and machine learning (ML). Although these machines take over tasks that have been executed by humans, they will not make human workforce obsolete. To leverage the potentials of $M L$, collaboration between humans and machines is necessary. Before collaboration processes can be developed, a classification of tasks in the field of ML is needed. Therefore, we present a taxonomy for the classification of tasks due to their complexity and the type of interaction. To derive insights about typical tasks and task-complexity, we conducted a literature review as well as a focus group workshop. We identified three levels of task-complexity and three types of interactions. Connecting them reveals three generic types of tasks. We provide prescriptive knowledge inherent in the task/interaction-taxonomy.
\end{abstract}

Keywords: Machine Learning, Task, Interaction, Collaboration, Crowdsourcing, Taxonomy

\section{Introduction}

To enhance performance and create opportunities for increased productivity, more and more companies implement innovations in artificial intelligence (AI) and machine learning (ML) with the aim of increasing automation of their business processes (Manyika et al. 2017). Recent breakthroughs in these fields, demonstrated for example by the computer program AlphaGo which was able to defeat several human professional players in the complex board game Go have extended the application range of machines in 
business as well as daily life. As a consequence, machines are more and more able to take over tasks that have previously been executed by humans. This development is raising public concerns about the future of work. As reported by Reuters (Reuters 2016), four out of ten young people believe that they will lose their jobs to machines. However, smart machines will not make human workforce obsolete. To fully leverage the potentials of AI and ML (e.g. new opportunities for added value like greater productivity, higher quality of results, new products and services), collaborative work between humans and machines is needed (Bahle et al. 2016). This implies that many humans will in future work alongside rapidly learning machines (Manyika et al. 2017). From that point of view, humans will not be replaced by machines, but current working forms for humans will change, as work activities will be reallocated and collaboration between humans and machines is needed. Therefore, the challenge will be to achieve an understanding of typical tasks for machines and for humans, and to develop techniques that will support collaboration between humans and machines and among themselves. Up to now, it is not clear how collaborative processes have to look like in order to effectively and efficiently shape collaboration between interacting humans and systems.

Combining techniques from the field of information systems (IS) research with techniques from disciplines concerned with designing collaborative processes among humans like Collaboration Engineering (Briggs et al. 2015) or Crowdsourcing (Durward et al. 2016) seems to be a promising solution. Applying an interdisciplinary approach has the potential to add a socio-technical perspective to include insights and competences of humans and machines equally. However, before designing collaborative processes, there is the necessity to be able to analyze and categorize tasks that exist in this context. This will pave the way towards the development of suitable process designs that will ensure an integration of competences of humans as well as machines according to their strengths. This raises the following research question.

RQ: How can tasks in the context of ML be classified based on their complexity and their type of interaction?

Therefore, the aim of this paper is to develop a taxonomy capable of classifying tasks based on their complexity and type of interaction. This taxonomy will serve as decision basis for developing suitable designs of collaboration. Furthermore, the taxonomy will outline demands to design collaboration as reusable work practices to leverage the potentials of ML.

\section{Methodological Approach}

In this paper, we use Design Science Research (DSR) and the according understanding of theory in DSR as a research framework. We structure our paper along Hevner's (Hevner 2007) three-cycle view (Figure 1). First, we start the relevance cycle by identifying a set of unsolved problems inherent in the development of learning cyber-physical systems that demand a classification of tasks and interaction types (Section 'Introduction'). Second, we initiate the rigor cycle by drawing on justificatory knowledge from AI, ML and Active Learning (AL) literature as well as collaboration literature (Section 'Theoretical and Conceptual Background'). To inform our taxonomy, we report the procedures and results of a literature analysis (Section 'Typical Types of Tasks in ML Literature') and report the procedures and results of a focus group workshop to derive insights concerning the origins of complexity in tasks (Section 'Origins of TaskComplexity in the Field of ML'). Thirdly, we completed a design cycle and report our taxonomy (Section 'Taxonomy of Tasks and Interaction Types'). Fourth, we describe possibilities for further research as well as limitations of the current study (Section 'Limitations and Future Research'). Fifth, we complete the rigor cycle by adding prescriptive knowledge ${ }^{1}$ (Gregor und Hevner 2013) to the literature and close with a conclusion (Section 'Contribution and Conclusion').

Referring to the contribution of our research, our solution, the "Task/Interaction Taxonomy", represents prescriptive knowledge that contributes towards a "theory for design and action" according to Gregor

${ }^{1}$ Prescriptive knowledge describes artifacts designed by humans to improve the natural world. It is inherent in the form of models, methods, instantiations, and design theories (Gregor and Hevner 2013)

2 Design Science Research distinguishes between five types of theory: "theory for analyzing" (says what is); "theory for explaining" (says what is, how, why, when, and where); "theory for predicting" (says what is and what will be); "theory for explaining and predicting" (says what is, how, why, when, where, and what will be); "theory for design and action" (says how to do something) (Gregor 2006) 
(2006). We inter alia depict justificatory knowledge from AI, ML, AL and CE literatures, and describe principles of form and function inherent in our taxonomy. The taxonomy is suitable to classify tasks in the context of ML due to their complexity and type of interaction.

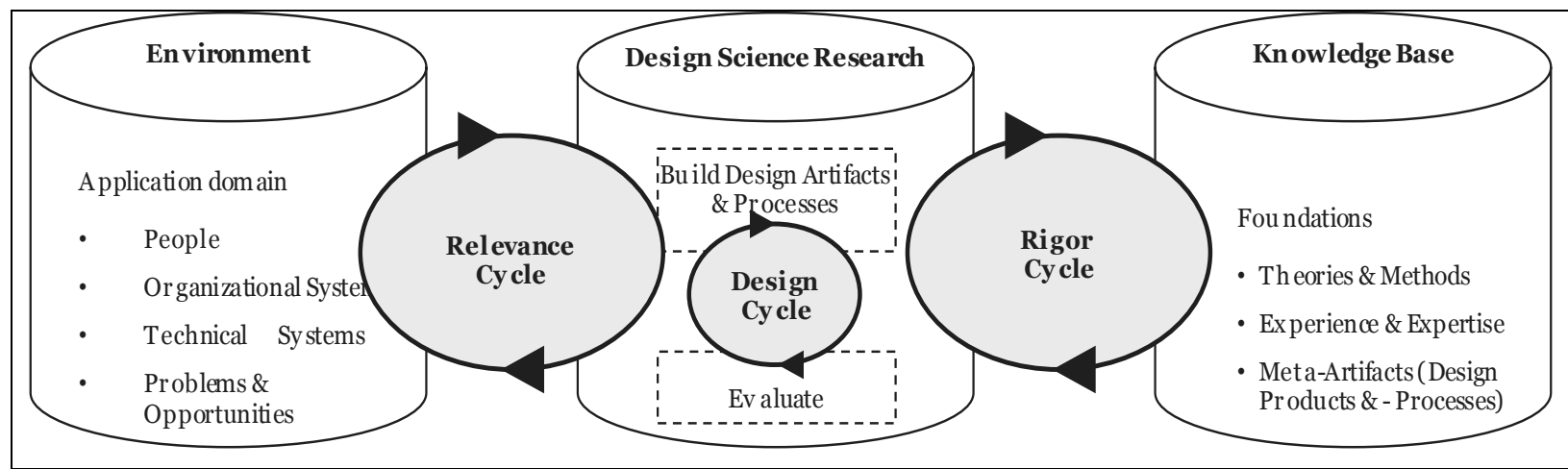

Figure 1. DSR Three Cycle View (Hevner 2007)

\section{Theoretical and Conceptual Background}

\section{Artificial Intelligence, Machine Learning and Active Learning}

AI is a field of computer science. We use the term in the sense of the automation of "activities that we associate with human thinking, activities such as decision-making, problem solving, learning" (Russell and Norvig 1995). In this sense, AI is the generic term that encompasses a range of approaches and techniques including concepts we use in this paper. One of these approaches is the concept of ML. It aims at developing computer systems that can autonomously improve with increasing experience (Mitchell 2006). There are multiple approaches of how to establish ML, for example deep learning, Bayesian networks and AL. The approach of AL starts with plenty of unlabeled data and the active learning system repeatedly asks an oracle (usually a human expert) for labels and by this refines its current model. The aim of AL is to achieve performance that is comparable to a model trained on a set of completely labeled data while incurring labeling costs that are much lower (Calma et al. 2016). Figure 2 illustrates the relations between AI, ML and $\mathrm{AL}$, showing that $\mathrm{AL}$ is one approach (among others) of ML while AI is a more generic concept, encompassing both of them.

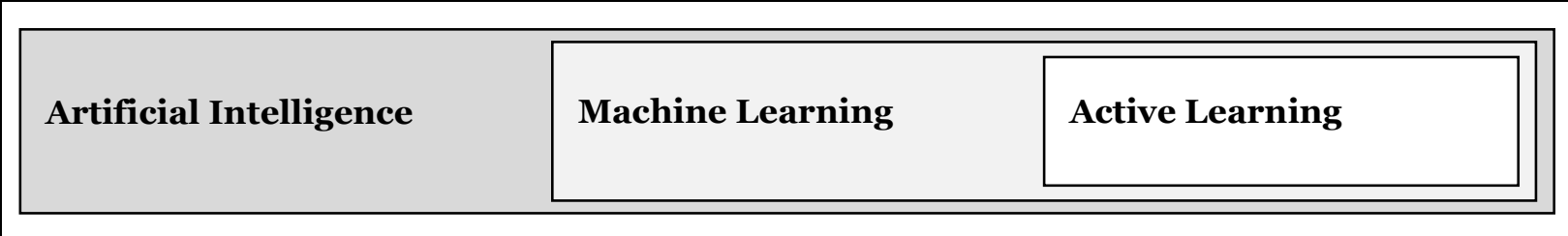

Figure 2. Overview of Artificial Intelligence, Machine Learning and Active Learning

\section{Collaboration in the Context of Machine Learning}

Using the term collaboration, we refer to the discipline of Collaboration Engineering (CE) which aims at "designing recurring collaboration processes that can be transferred to groups of humans that can be selfsustaining in these processes using collaboration techniques and technology" (Vreede und Briggs 2005). In this context, groups of humans can solve tasks collaboratively by engaging in a previously designed and validated sequence of collaborative activities. In such a process design, so called "patterns of collaboration" are used to structure collaborative activities. (Briggs et al. 2014). Table 1 shows and describes these five basic patterns of collaboration. 
Table 1. Patterns of Collaboration (Briggs et al. 2014)

\begin{tabular}{|l|l|}
\hline Pattern & Description \\
\hline 1. Diverge & Activities that lead from fewer concepts to more concepts. \\
\hline 2. Converge & $\begin{array}{l}\text { Activities that lead from a state of having many concepts to a focus on few but } \\
\text { promising concepts. }\end{array}$ \\
\hline 3. Organize & $\begin{array}{l}\text { Activities that lead to a greater understanding of the relationships among } \\
\text { concepts. }\end{array}$ \\
\hline 4. Evaluate & $\begin{array}{l}\text { Activities that lead to a greater understanding of the possible consequences of } \\
\text { concepts. }\end{array}$ \\
\hline 5. Build Consensus & Activities that lead to having more agreement on courses of action. \\
\hline
\end{tabular}

Furthermore, approaches from the discipline of Crowdsourcing can be useful. Crowdsourcing refers to an open call to an anonymous crowd with potential contributors that solve a task (Durward et al. 2016). Those approaches inter alia include collaborative peer-production (Brabham 2008) and can be referred to as an "online, distributed problem-solving and production model" (Brabham 2008).

\section{Typical Types of Tasks in Machine Learning Literature}

To derive an understanding of different typical tasks in this context and to achieve comprehension for how these tasks can be categorized, we conducted a literature review. We searched five major databases to identify relevant ML literature. We focused on title, abstract and keywords and used the search terms "complex task" and "machine learning", as it turned out that using other terms (e.g. "active learning") led to unsatisfactory and/or ambivalent results (e.g. literature from disciplines other than the researched). The search led to 868 hits, divided between the five queried databases. Table 2 provides a review of queried databases and shows respective hints.

Table 2. Summary of Queried Databases and Respective Hints

\begin{tabular}{|l|l|l|l|l|l|}
\hline Database & AIS Library & IEEE explore & ACM DL & Emerald Insight & Sciencedirect \\
\hline Hits & 2 & 396 & 223 & 7 & 240 \\
\hline
\end{tabular}

We analyzed the identified literature as follows: First, we filtered out papers that did not contain specific information about tasks. As most of the analyzed papers rather focus on the design of specific technical issues, this resulted in 27 papers that we subsequently analyzed in great detail. Second, we extracted the discussed task for each of these 27 papers and third, we classified the tasks to circumscribing categories. To derive those categories, we searched for similarities in tasks (e.g. application areas of the described techniques like text processing or image recognition). Table 3 shows the formed categories and the number of assigned papers as well as prompts describing the discussed tasks.

Our literature analysis reveals three basic categories. Tasks summed up under the category of 'Natural Language Processing' are concerned with text analysis like for example extracting knowledge from textual data in biomedical literature (Khalid and Sezerman 2017) as well as tasks of spoken language processing (e.g. in customer care applications) (Tur et al. 2003; Tur et al. 2005). The second category 'Image/Object Recognition' is made of tasks concerned with recognition of images and objects reaching from handwritten digits (Silva and Zhao 2015) to teaching a robot symbols (Kulick et al. 2013). Finally, a third category named 'Various Tasks' was established to sum up tasks that do not fit into the two previously formed groups. This category contains a range of tasks like extracting rhythmic patterns from music (Coca and Zhao 2016) or solving geotechnical engineering problems (Chou and Thedja 2016). Concluding from the literature review, the two most prominent types of tasks in literature are concerned either with NLP or the recognition of images and objects. 


\begin{tabular}{|c|c|c|c|}
\hline \multicolumn{4}{|c|}{ Table 3. Summary of Identified Tasks and Assigned Papers } \\
\hline Name of Category & $\begin{array}{l}\text { Natural Language } \\
\text { Processing (NLP) }\end{array}$ & $\begin{array}{l}\text { Image/Object } \\
\text { Recognition }\end{array}$ & Various Tasks \\
\hline $\begin{array}{l}\text { Number of } \\
\text { Assigned Papers }\end{array}$ & 11 & 8 & 8 \\
\hline Assigned Papers & $\begin{array}{l}\text { Chu et al. 2011: Analysis } \\
\text { of written comments } \\
\text { Druck et al. 2009: } \\
\text { Textual data analysis } \\
\text { Fehrer and Feuerriegel } \\
\text { 2015: Textual data } \\
\text { analysis } \\
\text { Giatsoglou et al. 2017: } \\
\text { Textual data analysis } \\
\text { Hakkani-Tür et al. } \\
\text { 2006: Spoken language } \\
\text { analysis } \\
\text { Hammerton et al. 2002: } \\
\text { Analysis of syntactic } \\
\text { structures } \\
\text { Kadhim and Omar 2012: } \\
\text { Textual data analysis } \\
\text { Khalid and Sezerman } \\
\underline{\text { 2017: Textual data }} \\
\text { analysis } \\
\text { Paquet et al. 2010: } \\
\text { Textual data analysis } \\
\text { Tur et al. 2005: Spoken } \\
\text { language analysis } \\
\text { Tur et al. 2003: Spoken } \\
\text { language analysis }\end{array}$ & $\begin{array}{l}\text { Joshi et al. 2012: Image } \\
\text { recognition } \\
\text { Kulick et al. 2013: Symbol } \\
\text { recognition } \\
\text { Narr et al. 2016: Object } \\
\text { recognition } \\
\text { Pronobis et al. 2008: } \\
\text { Object recognition } \\
\text { Silva and Zhao 2015: } \\
\text { Recognition of } \\
\text { handwritten digits } \\
\text { Vatsavai 2012: Image } \\
\text { recognition } \\
\text { Xiao et al. 2014: Image } \\
\text { recognition } \\
\text { Zheng et al. 2017: Image } \\
\text { recognition }\end{array}$ & $\begin{array}{l}\text { Chou and Thedja 2016: } \\
\text { Geotechnical } \\
\text { engineering problems } \\
\text { Coca and Zhao 2016: } \\
\text { Rhythm recognition } \\
\text { He et al. 2016: Fault } \\
\text { detection } \\
\text { Leung et al. 2014: Graph } \\
\text { structure analysis } \\
\text { Li et al. 2016: Fault } \\
\text { detection } \\
\text { Roggen et al. 2013: } \\
\text { Recognition of sensor } \\
\text { data } \\
\text { Sohail and Khanum } \\
\text { 2008: Complex network } \\
\text { management } \\
\text { Zhou et al. 2017: } \\
\text { Analyzing big data }\end{array}$ \\
\hline
\end{tabular}

However, looking at the results of the literature review, the tasks represent more or less some kind of machine-machine-collaboration. Since innovations in AI and ML will have the potential to change the future of work, we concluded that an additional perspective is needed to include tasks in the context of human-machine-collaboration. In CE, people make use of sequences of different activities of collaboration to solve complex tasks. Likewise, solving complex tasks by human-machine-collaboration can involve several activities which may inter alia include NLP and the recognition of images and/or objects. For example, a robot that is able to collaborate with humans may have to be able to understand spoken language and autonomously interact with its environment (which involves some sort of object recognition). Therefore, we assume that a new way of categorizing tasks in this context is needed. Thus, we suggest to categorize tasks by their inherent complexity and the type of interaction between humans and machines.

\section{Origins of Task-Complexity in the Field of ML}

As our aim is to distinguish between tasks according to their inherent complexity and matching them to types of interaction, the next step of our research activities was to identify origins of task-complexity in this field. In order to acquire the needed understanding of complexity in tasks, we carried out a focus group workshop. The workshop consisted of seven senior researchers coming from the disciplines of intelligent embedded systems, information systems and knowledge processing. During the workshop, the participants brainstormed about origins of complexity in tasks, resulting in 41 contributions. Subsequently, all participants presented their contributions to the group in order to achieve clarity. To analyze the data, we 
followed a qualitative content analysis according to Kohlbacher (Kohlbacher 2006). We documented the results of this activity, aggregated duplicates and overlaps and classified the named origins of complexity into four categories. Table 4 illustrates the four categories and its origins of task-complexity.

\begin{tabular}{|c|c|}
\hline Name of Category & Assigned Origins of Task-Complexity \\
\hline $\begin{array}{l}\text { Composition of } \\
\text { Interacting Entities }\end{array}$ & $\begin{array}{ll}\text { - } & \text { Quantity of interacting entities } \\
\text { - } & \text { Degree of heterogeneity of interacting entities } \\
\text { - } & \text { Required degree of expertise of interacting entities } \\
\text { - } & \text { Unknown expertise of interacting entities }\end{array}$ \\
\hline Communication Quality & $\begin{array}{l}\text { - } \text { Non-conformity of query answers } \\
\text { - } \quad \text { Error ratio } \\
\text { - } \quad \text { Effort and reliability of communication }\end{array}$ \\
\hline $\begin{array}{l}\text { Interaction } \\
\text { Language/Encoding }\end{array}$ & $\begin{array}{ll}\text { - } & \text { Interaction on different abstraction levels } \\
\text { - } & \text { Diverging vocabularies } \\
\text { - } & \text { Complex grammar and syntax }\end{array}$ \\
\hline Duration of Interaction & $\begin{array}{l}\text { - } \quad \text { Number of needed interactions } \\
\text { - } \quad \text { Length of interactions } \\
\text { - } \quad \text { Time until query response }\end{array}$ \\
\hline
\end{tabular}

After developing the categories, we interpreted the results and exploratively derived dependencies among them. This resulted to our following underlying research assumptions:

- Research Assumption 1: The number of needed interactions (i.e. 'Duration of Interaction') is dependent on the number and the expertise of interacting systems and/or humans (i.e. 'Composition of Interacting Entities').

- Research Assumption 2: The number of used abstraction levels in communication (i.e. 'Interaction Language/Encoding') is dependent on the heterogeneity of interacting entities (i.e. 'Composition of Interacting Entities').

- Research Assumption 3: The inconsistency of query answers (i.e. 'Communication Quality') is dependent on the heterogeneity of interacting entities (i.e. 'Composition of Interacting Entities').

Figure 3 visualizes the research assumptions and the identified dependencies between the established categories.

\begin{tabular}{|c|c|}
\hline \multicolumn{1}{|c|}{\begin{tabular}{c|c|}
\hline Composition of \\
Interacting Entities
\end{tabular}} \\
\hline $\begin{array}{c}\text { Duration of } \\
\text { Interaction }\end{array}$ & $\begin{array}{c}\text { Interaction } \\
\text { Language/Encoding }\end{array}$ \\
\hline Figure 3. Influences between Categories of Complexity \\
\hline
\end{tabular}

We identified the category 'Composition of Interacting Entities' as the determining factor for the three remaining categories. Origins of complexity that were classified into these three categories are rather consequences of factors situated in the category 'Composition of interacting Entities' than origins for complexity on their own. We therefore conclude that task-complexity in ML is mainly determined by the composition of interacting entities (e.g. number, nature and what is already known about them). Hence, we 
use the composition of interacting entities as the main factor for characterizing task-complexity. This will automatically include the subordinated categories.

\section{Taxonomy of Tasks and Interaction Types}

After surveying typical tasks in the field of ML with our literature analysis and identifying origins of taskcomplexity with our focus group workshop, we used these insights to develop a taxonomy (see Table 5). Our literature analysis illustrates the state of the art, whereas the results from the focus group workshop illustrate origins of task complexity. Since we aim to create a framework that has the potential to sketch opportunities of the future of work among the interplay of humans and machines, we needed to adjust our findings and created a taxonomy on a more abstract level. This taxonomy allows classifying tasks among several degrees of complexity and several types of interaction. Therefore, we combined the insights of our literature analysis and our focus group workshop. Against that background, the types of tasks identified in literature mainly refer to machine-machine interaction and, thus, are mainly inherent in the knowledge exploitation tasks that we identified in the taxonomy. The categories of task-complexity that resulted from the focus group workshops provided important foundations in the process to derive a more abstract definition of levels of task-complexity in the taxonomy.

Based on this, we identified three generic levels of task-complexity and three types of interaction (see Table 5). This way, the taxonomy classifies tasks due to their complexity and the type of interaction that is used to cope with this task. Since the three interaction types also refer to increasing complexity of collaborative activities, the taxonomy provides a guide for choosing the appropriate interaction type depending on the level of task complexity. Matching the degrees of task-complexity with the appropriate type of interaction, we identified three generic representative types of tasks in the cells of the table. Table 5 shows the developed taxonomy.

\begin{tabular}{|c|c|c|c|c|}
\hline \multicolumn{5}{|c|}{ Table 5. The "Task/Interaction" Taxonomy } \\
\hline & & \multicolumn{3}{|c|}{ Interaction Types } \\
\hline & & Machine-Machine & Machine-Human & Human-Human \\
\hline \multirow{3}{*}{$\begin{array}{l}\text { Levels of } \\
\text { Task- } \\
\text { Complexity }\end{array}$} & $\begin{array}{l}\text { Low Complexity } \\
\text { Task }\end{array}$ & $\begin{array}{c}\text { Knowledge } \\
\text { Exploitation Tasks }\end{array}$ & \multicolumn{2}{|c|}{ Area of Unexploited Potential } \\
\hline & $\begin{array}{l}\text { Average Complexity } \\
\text { Task }\end{array}$ & & $\begin{array}{c}\text { Knowledge } \\
\text { Validation Tasks }\end{array}$ & \\
\hline & $\begin{array}{l}\text { High Complexity } \\
\text { Task }\end{array}$ & \multicolumn{2}{|c|}{ Area of Arising Questions } & $\begin{array}{c}\text { Knowledge } \\
\text { Creation Tasks }\end{array}$ \\
\hline
\end{tabular}

The vertical axis of the taxonomy represents different 'Levels of Task-Complexity'. We distinguish between three basic levels of task-complexity, which are in hierarchical order.

Low Complexity Task: This type describes a situation in which machines can refer to a correct solution space or database. Therefore, they will be able to develop the correct solution on their own. Thus, we conclude that machines do not need humans to solve tasks of this type. As in these cases the group of interacting entities will be rather small and homogeneous, we assume these tasks to be of low complexity.

Average Complexity Task: Concerning these tasks, machines can refer to a solution space. However, solutions need to be verified by oracles (usually human experts) before they can be used. As solving these tasks normally needs the input of humans, they can be considered more complex than the group of tasks described before. Therefore, we assume these tasks to be of average complexity.

High Complexity Task: This category encompasses situations in which machines have to build a correct solution space as a basis first. Therefore, they need humans to propose a solution and create new knowledge. A process that needs collaboration between multiple humans. The solutions need to be verified by other humans before they can be accepted as correct. Thus, human-human interaction is needed to solve this type 
of task. As numerous and heterogeneous interacting entities with rather high degrees of expertise will be needed we assume these tasks to be of high complexity.

The horizontal axis represents the three 'Interaction Types'. We distinguish between three types, which are in hierarchical order. Depending on the composition of interacting entities, complexity increases with collaborative activities, and thus, along the interaction types.

Machine-Machine Interaction: For these kind of interactions, no humans are needed. Machines interact autonomously to solve given tasks.

Machine-Human Interaction: Interactions of this type are characterized by interactions between humans and machines. Involved humans typically do not interact among each other.

Human-Human Interaction: In this type of interaction, multiple humans are needed in order to be able to find the correct solution for a task. These humans have to collaborate with each other to develop the needed information as well as to verify possible solutions.

Matching the degrees of task-complexity with the interaction types, we identified three 'Generic Types of Tasks' that are defined by its level of task complexity and the interaction type.

- Knowledge Exploitation Tasks: For this kind of tasks there is already a fully and correctly labeled pool of data available that machines can refer to in order to develop a solution. These tasks are defined by a 'Low Complexity Task' and a 'Machine-Machine Interaction'.

- Knowledge Validation Tasks: This category consists of tasks that are typically concerned with validating collected information (e.g. labeling data and verifying current labels). As an input of oracles (e.g. human experts) is needed, we classify them as 'Average Complexity Tasks' for which a 'MachineHuman Interaction' is needed.

- Knowledge Creation Tasks: For this type of tasks, multiple humans typically collaborate to create knowledge to establish a solution space for machines to access. Therefore, we categorize them as 'High Complexity Tasks' for which a 'Human-Human Interaction' is needed.

Apart from these three generic types, we identified two areas in the taxonomy that require further explanation. Tasks that can be classified into the 'Area of Unexploited Potential' may offer potential for increased automation. For example, a Low Complexity Task that is solved by a Machine-Human Interaction may be fraught with design problems and bear potential to shift it further to the left, into the domain of a Machine-Machine-Interaction. Imagine for instance current driver assistance systems in cars that recognize speed limit signs and communicate them visually to a human driver. Shifting these interactions into the domain of a Machine-Machine Interaction, where the car recognizes signs and changes its speed autonomously represents a step towards the development of self-driving cars.

Some of the tasks that can be classified into the 'Area of Arising Questions' may also offer potential for increased automation. However, further automation of these tasks may often come with moral and legal concerns and can also raise questions of social acceptance. Imagine a High Complexity Task that is solved by a Machine-Human Interaction. This is especially the case when algorithms are designed to prepare, support and influence human decision making. An example is Equivant's algorithm that predicts the probability of recidivism of convicts to support the decisions of judges. According to a recent report (Angwin et al. 2017), the algorithm wrongly predicted a high risk of relapse twice as frequent for African-Americans than for whites. Another example is a future algorithm that autonomously judges candidates for a job and hires the one that fits best. It provides a useful example for a High Complexity Task that is solved by a Machine-Machine Interaction. Using such an algorithm raises questions of social acceptance, as employers may want to base their decisions on their knowledge of human nature, intuition or their experience rather than on statistic probabilities.

By matching different levels of complexity of tasks to interaction types, the taxonomy illustrates three generic types of tasks in the field of ML based on their complexity and types of interaction. Furthermore, it can help to identify tasks that are fraught with design problems and offer potential for further automation. Finally, it can be used to detect tasks where further automation may raise questions of social acceptance and/or moral and legal concerns. 


\section{Limitations and Future Research}

Regarding the limitations of our study, we note that the number of participants of the focus group workshop seems to be rather small. Since this field of research is rather new, explorative research is needed. Therefore, we chose a qualitative approach and selected participants that were experts in the field and started with this sample size. Even if qualitative studies are typically characterized by a smaller population, compared to quantitative studies, the taxonomy will be re-validated.

To take the next step for future research, we enlarged the sample of experts from the initial focus group workshop. We are currently cooperating with well-known scientists from the fields of intelligent embedded systems, information systems and knowledge processing. To acquire expert knowledge, we will conduct a Delphi study. In this study, the experts will first review the formed categories of task-complexity. Second, we will elaborate opportunities for expanding the taxonomy. For this purpose, the experts will use the taxonomy in order to classify tasks from their AI and ML research. Third the experts will brainstorm possible new tasks for the future of work to derive promising research opportunities. Against that background, this validation procedure seems to be appropriate since it provides several additional advantages. It creates a larger pool of typical task examples for the several cells of the taxonomy, which in turn will trigger discussions for future research and guidance for classifying tasks.

Besides that, we work with experts from practice and research and create a case study for each of the three 'Generic Types of Tasks' (Table 5) and design and evaluate the required and occurring collaborative activities among humans and machines. This will help to create the foundations for typical techniques to cope with various forms of work resulting from the levels of task-complexity and the interaction types. Those techniques inter alia focus on designing re-usable designs of collaborative activities that will support the interacting entities in solving a task. In this context, we currently run a crowdsourcing campaign and implemented a design of a collaborative process to gain insights for the case of the 'Knowledge Validation Task'.

\section{Contribution and Conclusion}

This paper contributes towards a 'theory of design and action' and inter alia provides prescriptive knowledge (Gregor 2013) in the form of a taxonomy. The developed taxonomy opens possibilities of classifying current as well as arising tasks in the field of ML based on their complexity and their types of interaction. It can be used as a basis for identifying tasks for which designing collaboration processes will be useful to set the basis for the interplay of humans and machines.

We conclude, that in near future, human workforce will not be replaced by machines. However, we discussed that the development of AI (e.g. learning systems) will fundamentally transform working environments by rearranging value-adding processes. In the face of this development, shaping collaboration between humans and machines is needed. A taxonomy for the classification of tasks in the field of ML opens the possibility to identify situations that require the development of these collaboration processes. In this paper, we identified origins of task-complexity that allowed us to distinguish between three different levels of task-complexity. We then linked these findings to interaction types, leading to the development of a taxonomy for the classification of tasks in the field of ML.

\section{Acknowledgement}

This paper presents research that was conducted in the context of the projects "CIL" and "StaySmart". CIL is funded by the University of Kassel (funding program for further profiling of the university 2017-2022: "Zukunft 2017-Standard"). StaySmart is funded by the German Federal Ministry of Education and Research (BMBF) (funding number: 02L12A170) and managed by the Project Management Agency Karlsruhe (PTKA). The responsibility for the content of this publication remains with the authors.

\section{References}

Angwin, Julia; Larson, Jeff; Kirchner, Lauren; Mattu, Surya: Machine Bias - ProPublica. Online accessible on https://www.propublica.org/article/machine-bias-risk-assessments-in-criminal-sentencing, last accessed on 09.03.2017. 
Bahle, Gernot; Calma, Adrian; Leimeister, Jan Marco; Lukowicz, Paul; Oeste-Reiss, Sarah; Reitmaier, Tobias et al. (2016): Lifelong Learning and Collaboration of Smart Technical Systems in Open-Ended Environments -- Opportunistic Collaborative Interactive Learning. In: 2016 IEEE International Conference on Autonomic Computing (ICAC). 2016 IEEE International Conference on Autonomic Computing (ICAC). Wuerzburg, Germany: IEEE, p. 315-324.

Brabham, Daren C. (2008): Crowdsourcing as a model for problem solving: An introduction and cases. In: Convergence 14 (1), p. 75-90.

Briggs, Robert O.; Kolfschoten, Gwendolyn L.; Vrede de GJ, Albrecht C; Lukosch, S.; Dean, D. L. (2015): A six-layer model of collaboration. In: Collaborative Systems: Concept, Value, and Use. Routledge, Taylor \& Francis Group, London, p. 211-227.

Briggs, Robert O.; Vreede, Gert-Jan de; Nunamaker Jr, Jay F. (2014): Collaboration Engineering with ThinkLets to Pursue Sustained Success with Group Support Systems. In: Journal of Management Information Systems 19 (4), p. 31-64.

Calma, A.; Leimeister, J. M.; Lukowicz, P.; Oeste-Reiß, S.; Reitmaier, T.; Schmidt, A. et al. (2016): From Active Learning to Dedicated Collaborative Interactive Learning. In: 4th International Workshop on Self-Optimisation in Autonomic and Organic Computing Systems (SAOS).

Chou, Jui-Sheng; Thedja, Julian Pratama Putra (2016): Metaheuristic optimization within machine learning-based classification system for early warnings related to geotechnical problems. In: Automation in Construction 68, p. 65-80.

Chu, Wei; Zinkevich, Martin; Li, Lihong; Thomas, Achint; Tseng, Belle (2011): Unbiased online active learning in data streams. In: Proceedings of the 17th ACM SIGKDD international conference on Knowledge discovery and data mining. ACM, p. 195-203.

Coca, Andrés E.; Zhao, Liang (2016): Musical rhythmic pattern extraction using relevance of communities in networks. In: Information Sciences 329, p. 819-848.

Druck, Gregory; Settles, Burr; McCallum, Andrew (2009): Active Learning by Labeling Features. In: Proceedings of the 2009 Conference on Empirical Methods in Natural Language Processing: Volume 1 - Volume 1. Stroudsburg, PA, USA: Association for Computational Linguistics (EMNLP '09), p. 81-90.

Durward, David; Blohm, Ivo; Leimeister, Jan Marco (2016): Crowd Work. In: Business \& Information Systems Engineering (BISE) 58 (4), p. 281-286.

Fehrer, Ralph; Feuerriegel, Stefan (2015): Improving Decision Analytics with Deep Learning: The Case of Financial Disclosures. In: arXiv preprint arXiv:1508.01993.

Giatsoglou, Maria; Vozalis, Manolis G.; Diamantaras, Konstantinos; Vakali, Athena; Sarigiannidis, George; Chatzisavvas, Konstantinos Ch. (2017): Sentiment analysis leveraging emotions and word embeddings. In: Expert Systems with Applications 69, p. 214-224.

Gregor, S. (2006): The Nature of Theory in Information Systems. In: MIS quarterly 30 (3), S. 611-642.

Silva, Thiago Christiano; Zhao, Liang (2015): High-level pattern-based classification via tourist walks in networks. In: Information Sciences 294, p. 109-126.

Gregor, S.; Hevner, A. R. (2013): Positioning and Presenting Design Science Research for Maximum Impact. In: MIS Quarterly (MISQ) 37 (2), p. 337-355.

Hakkani-Tür, Dilek; Riccardi, Giuseppe; Tur, Gokhan (2006): An active approach to spoken language processing. In: ACM Transactions on Speech and Language Processing (TSLP) 3 (3), p. 1-31.

Hammerton, James; Osborne, Miles; Armstrong, Susan; Daelemans, Walter (2002): Introduction to Special Issue on Machine Learning Approaches to Shallow Parsing. In: J. Mach. Learn. Res. 2, p. 551558.

He, Suowei; Wang, Zhiwei; Wang, Zhanwei; Gu, Xiaowei; Yan, Zengfeng (2016): Fault detection and diagnosis of chiller using Bayesian network classifier with probabilistic boundary. In: Applied Thermal Engineering 107, p. 37-47.

Hevner, Alan R. (2007): A three cycle view of design science research. In: Scandinavian journal of information systems 19 (2), p. 4.

Joshi, Ajay J.; Porikli, Fatih; Papanikolopoulos, Nikolaos P. (2012): Scalable active learning for multiclass image classification. In: IEEE transactions on pattern analysis and machine intelligence 34 (11), p. 2259-2273.

Khalid, Zoya; Sezerman, Osman Ugur (2017): ZK DrugResist 2.0: A TextMiner to Extract Semantic Relations of Drug Resistance from PubMed. In: Journal of biomedical informatics.

Kohlbacher, Florian (2006): The use of qualitative content analysis in case study research. In: Forum Qualitative Sozialforschung/Forum: Qualitative Social Research, Bd. 7. 
Kulick, Johannes; Toussaint, Marc; Lang, Tobias; Lopes, Manuel (2013): Active Learning for Teaching a Robot Grounded Relational Symbols. In: IJCAI.

Leung, Carson Kai-Sang; MacKinnon, Richard Kyle; Wang, Yang (2014): A machine learning approach for stock price prediction: ACM.

Li, Dan; Zhou, Yuxun; Hu, Guoqiang; Spanos, Costas J. (2016): Fault detection and diagnosis for building cooling system with a tree-structured learning method. In: Energy and Buildings 127, p. 540-551.

M. H. Kadhim; N. Omar (2012): Automatic arabic Text Categorization using Bayesian learning. In: 2012 7th International Conference on Computing and Convergence Technology (ICCCT), p. 415-419.

Manyika, James; Chui, Michael; Lund, Susan; Ramaswamy, Sree (2017): WHAT'S NOW AND NEXT IN ANALYTICS, AI, AND AUTOMATION. Hg. v. McKinsey Global Institute, last accessed on 03.08.2017.

Mitchell, Tom Michael (2006): The discipline of machine learning: Carnegie Mellon University, School of Computer Science, Machine Learning Department (9).

Narr, Alexander; Triebel, Rudolph; Cremers, Daniel (2016): Stream-based Active Learning for efficient and adaptive classification of 3D objects. In: 2016 IEEE International Conference on Robotics and Automation (ICRA). 2016 IEEE International Conference on Robotics and Automation (ICRA). Stockholm, Sweden: IEEE, p. 227-233.

Paquet, Ulrich; van Gael, Jurgen; Stern, David; Kasneci, Gjergji; Herbrich, Ralf; Graepel, Thore (2010): Vuvuzelas \& active learning for online classification. In: NIPS Workshop on Comp. Social Science and the Wisdom of Crowds.

Pronobis, A.; Martinez Mozos, O.; Caputo, B. (2008): SVM-based discriminative accumulation scheme for place recognition. In: 2008 IEEE International Conference on Robotics and Automation. 2008 IEEE International Conference on Robotics and Automation (ICRA). Pasadena, CA, USA: IEEE, S. 522-529.

Reuters (2016): Many young people fear losing their jobs to robots, survey finds. Online accessible on http://in.reuters.com/article/davos-meeting-robots-survey-idINKCNoUWoG9, last accessed on 05.05.2017.

Roggen, D.; Troster, Gerhard; Lukowicz, P.; Ferscha, A.; del R. Millan, Jose; Chavarriaga, R. (2013): Opportunistic human activity and context recognition. In: Computer 46 (2), p. 36-45.

Sohail, S.; Khanum, A. (2008): Simplifying Network Management with Fuzzy Logic. In: ICC Workshops 2008 IEEE International Conference on Communications Workshops. ICC 2008 - 2008 IEEE International Conference on Communications Workshops. Beijing, China: IEEE, p. 195-201.

Tur, Gokhan; Hakkani-Tür, Dilek; Schapire, Robert E. (2005): Combining active and semi-supervised learning for spoken language understanding. In: Speech Communication 45 (2), S. 171-186.

Tur, Gokhan; Schapire, Robert E.; Hakkani-Tur, Dilek (2003): Active learning for spoken language understanding. In: Acoustics, Speech, and Signal Processing, 2003. Proceedings.(ICASSP'03). 2003 IEEE International Conference on, Bd. 1. IEEE, S. I.

Vatsavai, Ranga Raju (2012): A Machine Learning System for Recognizing Subclasses. In: Proceedings of the 3rd International Conference on Computing for Geospatial Research and Applications. New York, NY, USA: ACM (COM.Geo '12), p. 32:1-32:2.

Vreede, G-J de; Briggs, Robert O. (2005): Collaboration engineering: designing repeatable processes for high-value collaborative tasks. In: System Sciences, 2005. HICSS'05. Proceedings of the 38th Annual Hawaii International Conference on. IEEE, p. 17c-17c.

Xiao, Tianjun; Zhang, Jiaxing; Yang, Kuiyuan; Peng, Yuxin; Zhang, Zheng (2014): Error-Driven Incremental Learning in Deep Convolutional Neural Network for Large-Scale Image Classification. In: Kien A. Hua, Yong Rui, Ralf Steinmetz, Alan Hanjalic, Apostol (Paul) Natsev und Wenwu Zhu (Hg.): Proceedings of the ACM International Conference on Multimedia - MM '14. the ACM International Conference. Orlando, Florida, USA, 3/11/2014 - 7/11/2014. New York, New York, USA: ACM Press, p. 177-186.

Zheng, Yu; Fan, Jianping; Zhang, Ji; Gao, Xinbo (2017): Hierarchical learning of multi-task sparse metrics for large-scale image classification. In: Pattern Recognition 67, p. 97-109.

Zhou, Lina; Pan, Shimei; Wang, Jianwu; Vasilakos, Athanasios V. (2017): Machine learning on big data. Opportunities and challenges. In: Neurocomputing 237, p. 350-361. 